\title{
Pressão Arterial, Glicemia Capilar e Medidas Antropométricas em uma População Yanomámi
}

\section{Blood Pressure, Capillary Glucose, and Anthropometric Measurements in a Yanomámi Population}

\author{
Katia V. Bloch ${ }^{1}$; Evandro da S. F. Coutinho ${ }^{2}$; Maria Stella de C. Lôbo ${ }^{1}$; \\ José Egídio P. de Oliveira ${ }^{1} \&$ Adolfo Milech ${ }^{3}$
}

BLOCH, K. V.; COUTINHO, E. S. F.; LÔBO, M. E. C.; OLIVEIRA, J. E. P. \& MILECH, A.

Blood Pressure, Capilar Glucose and Anthropometric Measures in a Yanomámi Population. Cad. Saúde Públ., Rio de Janeiro, 9 (4): 428-438, oct/dec, 1993.

Seventy-two Yanomámi Indians from Surucucus, Roraima, Brazil, were examined to evaluate the correlation between capillary glucose level (CGL), systolic and diastolic blood pressure (SBP and $D B P$ ), and anthropometrical features. Blood pressure was higher among men than women: $S B P=109.8$ and $100.2 \mathrm{~mm} \mathrm{Hg} ; D B P=71.2$ and $63.5 \mathrm{mmHg}$, respectively. Levels compatible with hypertension were not found. SBP was correlated negatively with age and positively with height, weight, body mass index (BMI), and waist and hip circumferences. Controlling for BMI did not change the negative correlation between SBP and age. When weight was controlled, correlation between SBP and height changed to a negative value, but when height was controlled, positive correlation between weight and PAS was mantained. DBP was positively correlated with weight and height.

Capillary glucose level was significantly higher among women than men: 114.1 and $98.4 \mathrm{mg} / \mathrm{dl}$, respectively. When weight was controlled, waist circumference correlated positively with CGL. When waist circumference was controlled, weight and BMI correlated negatively with CGL. The results suggest that while blood pressure is correlated especially with body mass, capillary glucose level is more correlated with abdominal fat distribution.

Key words: Blood Pressure; Capillary Glucose; Anthropometric Measurements; Yanomámi

\section{INTRODUÇÃO}

Os Yanomámi constituem uma nação indígena que habita a floresta tropical na fronteira do Brasil e da Venezuela. No Brasil, estima-se que sejam cerca de 10.000 índios dispersos por mais

\footnotetext{
${ }^{1}$ Serviço de Saúde Coletiva do Hospital Universitário Clementino Fraga Filho (UFRJ). Avenida Brigadeiro Trompowsky, $s / n^{o}, 5^{\circ}$ andar, Ilha do Fundão, Rio de Janeiro, RJ, 21941-590, Brasil.

${ }^{2}$ Departamento de Epidemiologia e Métodos Quantitativos em Saúde da Escola Nacional de Saúde Pública. Rua Leopoldo Bulhões, 1480, $8^{\circ}$ andar, Rio de Janeiro, RJ, 21041-210, Brasil.

${ }^{3}$ Serviço de Nutrologia do Hospital Universitário Clementino Fraga Filho (UFRJ). Avenida Brigadeiro Trompowsky, $s / n^{o}, 9^{\circ}$ andar, Ilha do Fundão, Rio de Janeiro, RJ, 21941-590, Brasil.
}

de 150 malocas, em uma área aproximada de 9.418.000 hectares (Menegola \& Lôbo, 1991; Pithan et al., 1991a, 1991b). Dadas as suas características seminômades, os Yanomámi deslocam-se a cada 3-5 anos.

O suporte alimentar tradicional é oriundo da coleta, pesca, agricultura itinerante e caça, estas últimas representando cerca de $73 \%$ da ingestão protéica (Albert, 1985). Não há formas duradouras de armazenamento alimentar, sendo a atividade física desses índios proporcional às atividades laborativas necessárias à sua subsistência.

Sabe-se que os Yanomámi têm uma baixa ingestão de sódio, além de baixos índices de massa corporal (IMC) e de pressão arterial, esta última não se elevando com a idade (INTERSALT, 1988). 
Somente nos anos 50 tiveram início contatos mais permanentes entre os Yanomámi e a cultura ocidental, sobretudo com missionários e extrativistas. Entretanto, a grande invasão da área por garimpeiros tornou este contato mais intenso na década de 80 , trazendo uma série de doenças infecto-contagiosas (Pithan et al., 1991a, 1991b). O modus vivendi de alguns grupos Yanomámi vêm se modificando desde então, passando por um acelerado processo de sedentarização e mudança de hábitos alimentares. Estabeleceu-se, assim, uma dependência de alimentos que eram estranhos à dieta dos Yanomámi, como, por exemplo, o sal, o açúcar e outros produtos industrializados.

Estudos com populações primitivas que sofreram processos de aculturação têm demonstrado um aumento na prevalência de doenças cardiovasculares (DCV), diabetes e transtornos mentais (Dunn et al., 1970; Maculay et al., 1988; Montour \& Maculay, 1988; Zimmet et al., 1990; Musto, 1990; Hern, 1991). Tal quadro assume ainda maior gravidade se consideradas as dificuldades de populações primitivas, sobretudo no Brasil, quanto ao acesso ao sistema de saúde. Assim, torna-se imperativo avaliar e monitorar as variáveis ou fatores de risco que se identificam a partir do contato interétnico, a fim de se prevenir o aparecimento destas doenças.

Seguindo esta premissa, o objetivo deste trabalho é estudar o comportamento das pressões arteriais (sistólica e diastólica) e da glicemia capilar em função de medidas antropométricas em um grupo Yanomámi.

\section{MATERIAL E MÉTODOS}

Em fevereiro de 1992 foram examinados 72 índios Yanomámi na Área de Relações Intercomunitárias (ARI) de Surucucus, no Estado de Roraima, sendo a maioria proveniente de diferentes aldeias desta ARI. Os exames foram realizados em índios de ambos os sexos, com 18 ou mais anos de idade estimada. Dentre os 62 índios examinados no hospital de Surucucus, 17 estavam em tratamento para a malária, enquanto os outros 45 eram acompanhantes, estavam de passagem pelo local ou habitavam uma aldeia junto ao hospital. Estando este localizado no meio da selva, é bastante comum que os índios fiquem nos arredores do hospital por curtos períodos, mesmo sem estarem doentes ou acompanhando o tratamento de parentes. Dez índios foram examinados em outra aldeia próxima visitada na época.

\section{Coleta de Dados}

Os registros e as medidas foram realizados por um dos autores (Bloch), contando com a colaboração da equipe de saúde da unidade de Surucucus para a identificação dos índios e tradução das respostas.

Foram registrados o local de origem (aldeia), o nome atribuído, o sexo, a idade, a idade gestacional, a presença de patologias e o tempo de tratamento. Procedeu-se à aproximação da idade, que foi avaliada subjetivamente pela examinadora e pela equipe de saúde local, dada a ausência de registros de natalidade. Da mesma forma, a idade gestacional, medida por ciclos lunares, também foi estimada, quando a índia dispunha-se a falar no assunto. As patologias foram registradas quando diagnosticadas no hospital: malária (falciparum, vivax ou mista), anemia, desnutrição.

\section{Medidas}

As medidas realizadas foram as seguintes:

1. Pressão Arterial (PA), em mm Hg, medida com aparelho aneróide e manguito padrão para adultos. Foi registrada como pressão arterial sistólica (PAS) a fase I de Korotkof e como pressão arterial diastólica (PAD) a fase $\mathrm{V}$ de Korotkof. As medidas foram realizadas no braço direito, com os índios sentados, no início e no fim da avaliação, com cerca de 15 minutos de intervalo e antes da medida da glicemia. Utilizou-se a segunda medida na análise dos dados;

2. Glicemia Capilar, em $\mathrm{mg} / \mathrm{dl}$, avaliada no momento da entrevista, através de sangue capilar obtido por punção de polpa digital realizada com lanceta, com o auxílio de fita reagente (Glucostix - Laboratórios Miles do Brasil Ltda.) e de reflectômetro portátil (Glucometer II - Laboratórios Miles do Brasil Ltda.). O aparelho possuía mecanismo para a aferição periódica da calibragem; 
3. Peso, em quilos, aferido em balança tipo médica, para as medidas realizadas no hospital, e em balança portátil, para as medidas realizadas na aldeia. A diferença observada entre as balanças foi inferior a $1 \mathrm{~kg}$;

4. Altura, em metros, medida por antropômetro de balança (no hospital) e por antropômetro portátil (na aldeia), este último composto de uma prancheta de madeira com presilha, uma trena e um esquadro;

5. Circunferência Abdominal (CA), em milímetros, medida com fita métrica não-distensível, ajustada ao nível da cicatriz umbilical;

6. Circunferência do Quadril (CQ), em milímetros, medida com fita métrica não-distensível, ajustada ao nível do maior diâmetro do glúteo;

7. Temperatura Ambiente, em graus centígrados, medida através de termômetro ambiental colocado próximo ao local da avaliação.

Foram calculados o índice de massa corporal (IMC), pela fórmula peso $(\mathrm{kg}) /$ altura $(\mathrm{m})$, e a razão circunferência abdominal $(\mathrm{mm}) /$ circunferência do quadril (mm).

\section{Análise dos Dados}

Quatro índias gestantes foram excluídas da análise. Embora, em uma análise prévia, os resultados obtidos não tenham diferido de maneira importante com a inclusão ou exclusão dos 17 índios com malária, optou-se por não incluí-los nos resultados aqui apresentados.

Inicialmente foram feitas as estatísticas descritivas, isto é, as medidas de tendência central e de dispersão. Não foi o intuito desta etapa da análise estimar tais parâmetros objetivando inferências para toda a população Yanomámi, por não se tratar de uma amostra aleatória. Pretendeu-se definir os perfis antropométrico, pressórico e glicêmico do grupo estudado.

Devido ao tamanho da amostra, avaliou-se a distribuição das frequências, constatando-se que grande parte das variáveis não apresentava distribuição normal. Conseqüentemente, optouse pela utilização de testes não-paramétricos para comparações internas e para o estudo das correlações entre as variáveis.

A comparação das médias das variáveis antropométricas, idade, pressão arterial e freqüência cardíaca entre os sexos foi feita pela prova de Kruskal-Wallis (Siegel, 1981).

As correlações entre pressões arteriais (sistólica e diastólica) e as variáveis antropométricas foram estudadas através do coeficiente de correlação de postos de Spearmann. O controle de variáveis potencialmente confounding foi feito através do coeficiente parcial de Spearmann (Siegel, 1981).

\section{RESULTADOS}

A população estudada era composta de 29 homens $(56,9 \%)$ e 22 mulheres $(43,1 \%)$. As medidas de tendência central e de dispersão das variáveis idade, pressão arterial, glicemia e medidas antropométricas, por sexo, estão resumidas na Tabela 1. No grupo estudado, o peso, o IMC, a circunferência do quadril e a altura apresentaram médias maiores entre os homens. Já a circunferência abdominal e a relação cintura/quadril tiveram médias mais elevadas entre as mulheres.

\section{Pressão Arterial}

As médias das pressões arteriais, tanto a sistólica quanto a diastólica, foram mais altas nos homens. Não foram registrados valores compatíveis com o diagnóstico de hipertensão arterial, quer pelo critério da Organização Mundial da Saúde - 160 X 95 mm Hg (OMS, 1978), quer pelo do National Institute of Health - 140 X 90 mmHg (Joint National Committee, 1988).

Na Tabela 2 são apresentados os coeficientes de correlação de postos de Spearmann.

A PAS correlacionou-se inversamente com a idade e positivamente com peso, altura, circunferências abdominal e de quadril e IMC.

Quanto à PAD, apenas o peso e a altura correlacionaram-se positivamente. O coeficiente de correlação com a idade foi próximo a zero.

A análise das correlações revelou, ainda, que a idade relacionava-se inversamente com as variáveis antropométricas. Por este motivo, decidiu-se estimar as correlações entre níveis tensionais e variáveis antropométricas controlando-se fatores potencialmente confounding, através dos coeficientes parciais de correlação de Spearmann. Estes resultados encontram-se nas Tabelas 3 (PAS) e 4 (PAD). 
TA BE LA 1. Perfil das Variáveis Idade, Pressão Arterial, Glicemia Capilar e Medidas Antropométricas, por Sexo, no Grupo Estudado

\begin{tabular}{|c|c|c|c|}
\hline Variável & Feminino & Masculino & Total \\
\hline \multicolumn{4}{|l|}{ Idade (anos) } \\
\hline Número & 22 & 29 & 51 \\
\hline Média & 35,6 & 29,3 & 32,0 \\
\hline Desvio Padrão & 11,5 & 11,4 & 11,8 \\
\hline \multicolumn{4}{|l|}{ Peso $(\mathrm{Kg})$} \\
\hline Número & 21 & 28 & 49 \\
\hline Média & 41,8 & 49,7 & 42,5 \\
\hline Desvio Padrão & 4,9 & 3,9 & 5,8 \\
\hline \multicolumn{4}{|l|}{ Altura (cm) } \\
\hline Número & 22 & 29 & 51 \\
\hline Média & 1,43 & 1,52 & 1,48 \\
\hline Desvio Padrão & 0,04 & 0,04 & 0,06 \\
\hline \multicolumn{4}{|c|}{ Circunferência Abdominal (mm) } \\
\hline Número & 21 & 29 & 50 \\
\hline Média & 768,5 & 759,6 & 763,3 \\
\hline Desvio Padrão & 54,5 & 41,0 & 46,8 \\
\hline \multicolumn{4}{|c|}{ Circunferência do Quadril (mm) } \\
\hline Número & 22 & 29 & 51 \\
\hline Média & 807,5 & 817,9 & 813,4 \\
\hline Desvio Padrão & 43,0 & 24,7 & 33,8 \\
\hline \multicolumn{4}{|l|}{ PAS (mm Hg) } \\
\hline Número & 22 & 29 & 51 \\
\hline Média & 100,2 & 109,8 & 105,6 \\
\hline Desvio Padrão & 8,2 & 8,9 & 9,8 \\
\hline \multicolumn{4}{|l|}{$\mathrm{PAD}(\mathrm{mm} \mathrm{Hg})$} \\
\hline Número & 22 & 29 & 51 \\
\hline Média & 63,5 & 71,2 & 67,9 \\
\hline Desvio Padrão & 6,9 & 7,6 & 8,2 \\
\hline \multicolumn{4}{|l|}{ Glicose (mg/dl) } \\
\hline Número & 22 & 29 & 51 \\
\hline Média & 114,1 & 98,4 & $105,230,9$ \\
\hline Desvio Padrão & 28,2 & 31,6 & \\
\hline \multicolumn{4}{|l|}{$\mathrm{IMC}\left(\mathrm{kg} / \mathrm{m}^{2}\right)$} \\
\hline Número & 21 & 28 & 49 \\
\hline Média & 20,5 & 21,5 & 21,1 \\
\hline Desvio Padrão & 1,9 & 1,4 & 1,7 \\
\hline \multicolumn{4}{|l|}{$\mathrm{CA} / \mathrm{CQ}(\mathrm{mm})$} \\
\hline Número & 21 & 29 & 50 \\
\hline Média & 0,95 & 0,93 & 0,94 \\
\hline Desvio Padrão & 0,04 & 0,04 & 0,04 \\
\hline
\end{tabular}


TA B LA 2. Coeficientes de Correlação de Spearmann de Pressões Arteriais e Glicemia com Idade e Medidas Antropométricas

\begin{tabular}{llrrrrrr}
\hline \hline & & \multicolumn{1}{l}{ Idade } & Peso & Altura & $\begin{array}{c}\text { Circunferência } \\
\text { Abdominal }\end{array}$ & $\begin{array}{c}\text { Circunferência } \\
\text { do Quadril }\end{array}$ & IMC \\
\hline PAS & $\mathrm{a}$ & $-0,21$ & $+0,49$ & $+0,41$ & $+0,25$ & $+0,41$ & $+0,48$ \\
& $\mathrm{~b}$ & 0,14 & 0,00 & 0,00 & 0,00 & 0,00 & 0,00 \\
& $\mathrm{c}$ & 51 & 49 & 51 & 50 & 51 & 49 \\
\multirow{3}{*}{ PAD } & $\mathrm{a}$ & $+0,07$ & $+0,33$ & $+0,38$ & $+0,05$ & $+0,06$ & $+0,18$ \\
& $\mathrm{~b}$ & 0,61 & 0,02 & 0,01 & 0,70 & 0,65 & 0,22 \\
& $\mathrm{c}$ & 51 & 49 & 51 & 50 & 51 & 49 \\
Glicose & $\mathrm{a}$ & $+0,20$ & $-0,19$ & $-0,13$ & $+0,22$ & $+0,08$ & $-0,10$ \\
& $\mathrm{~b}$ & 0,15 & 0,19 & 0,36 & 0,12 & 0,60 & 0,51 \\
& $\mathrm{c}$ & 51 & 49 & 51 & 50 & 51 & 49 \\
\hline \hline
\end{tabular}

$\mathrm{a}=$ coeficiente de correlação de Spearmann $\quad \mathrm{b}=\mathrm{p}$-valor $\quad \mathrm{c}=$ número de observações

Alguns aspectos merecem ser destacados para uma posterior reflexão na discussão dos achados. O controle pelo IMC não alterou a correlação inversa que existia entre PAS e idade. Quando controlada pela circunferência abdominal, esta correlação inversa se intensificou.

A correlação positiva que existia entre a altura e a PAS inverteu-se quando se operou o controle pela variável peso. Já a associação da circunferência abdominal com a PAS enfraqueceu-se com o controle por esta mesma variável (peso).
No caso da PAD, sua correlação com a idade apresentou um discreto incremento quando controlada pelo IMC. Ao controlar-se pelo peso, a correlação entre PAD e altura se enfraqueceu.

\section{Glicemia Capilar}

Em relação à glicemia, as mulheres tiveram médias mais elevadas do que os homens (114,1 $\mathrm{mg} / \mathrm{dl}$ e $98,4 \mathrm{mg} / \mathrm{dl}$, respectivamente). Considerou-se que todas as glicemias foram medidas em períodos pós-prandiais, tendo sido encontrado um resultado superior a $200 \mathrm{mg} / \mathrm{dl}$.

TABELA 3. Coeficientes Parciais de Correlação de Spearmann e p-valor entre Pressão Sistólica e Medidas Antropométricas e Idade

\begin{tabular}{|c|c|c|c|c|c|c|c|}
\hline \multirow[b]{2}{*}{$\begin{array}{l}\text { Variável } \\
\text { de Controle }\end{array}$} & & \multicolumn{6}{|c|}{ Coeficientes de Correlação entre PAS e } \\
\hline & & Idade & Peso & Altura & $\begin{array}{c}\text { Circunferência } \\
\text { Abdominal }\end{array}$ & $\begin{array}{c}\text { Circunferência } \\
\text { do Quadril }\end{array}$ & IMC \\
\hline \multirow[t]{2}{*}{ Idade } & $\mathrm{a}$ & - & $+0,44$ & $+0,29$ & $+0,31$ & $+0,38$ & $+0,46$ \\
\hline & $\mathrm{b}$ & - & 0,00 & 0,05 & 0,03 & 0,01 & 0,00 \\
\hline \multirow[t]{2}{*}{ Peso } & a & $-0,10$ & - & $-0,10$ & $+0,26$ & $+0,33$ & $+0,46$ \\
\hline & $\mathrm{b}$ & 0,51 & - & 0,49 & 0,07 & 0,02 & 0,00 \\
\hline \multirow[t]{2}{*}{ Altura } & a & $-0,17$ & $+0,38$ & - & $+0,26$ & $+0,33$ & $+0,46$ \\
\hline & $\mathrm{b}$ & 0,26 & 0,01 & - & 0,07 & 0,02 & 0,00 \\
\hline \multirow[t]{2}{*}{ Circunferência Abdominal } & $\mathrm{a}$ & $-0,30$ & $+0,44$ & $+0,34$ & - & $+0,33$ & $+0,42$ \\
\hline & $\mathrm{b}$ & 0,04 & 0,00 & 0,02 & - & 0,02 & 0,00 \\
\hline \multirow[t]{2}{*}{ IMC } & a & $-0,19$ & $+0,26$ & $+0,30$ & $-0,03$ & $+0,16$ & - \\
\hline & $\mathrm{b}$ & 0,21 & 0,08 & 0,04 & 0,87 & 0,29 & - \\
\hline
\end{tabular}

$\mathrm{a}=$ coeficiente de correlação de Spearmann $\mathrm{b}=\mathrm{p}$-valor 
TA BELA 4. Coeficientes Parciais de Correlação de Spearmann e p-valor entre Pressão Diastólica e Medidas Antropométricas e Idade

\begin{tabular}{|c|c|c|c|c|c|c|c|}
\hline \multirow[b]{2}{*}{$\begin{array}{l}\text { Variável } \\
\text { de Controle }\end{array}$} & & \multicolumn{6}{|c|}{ Coeficientes de Correlação entre PAD e } \\
\hline & & Idade & Peso & Altura & $\begin{array}{c}\text { Circunferência } \\
\text { Abdominal } \\
\end{array}$ & $\begin{array}{c}\text { Circunferência } \\
\text { do Quadril }\end{array}$ & $\mathrm{IMC}$ \\
\hline \multirow[t]{2}{*}{ Idade } & $\mathrm{a}$ & - & $+0,38$ & $+0,36$ & $+0,06$ & $+0,07$ & $+0,20$ \\
\hline & $\mathrm{b}$ & - & 0,01 & 0,01 & 0,67 & 0,65 & 0,17 \\
\hline \multirow[t]{2}{*}{ Peso } & $\mathrm{a}$ & $+0,30$ & - & $+0,10$ & $-0,07$ & $-0,25$ & $-0,05$ \\
\hline & $\mathrm{b}$ & 0,17 & - & 0,52 & 0,64 & 0,10 & 0,72 \\
\hline \multirow[t]{2}{*}{ Altura } & $\mathrm{a}$ & $+0,18$ & $+0,12$ & - & $+0,05$ & $-0,08$ & $+0,13$ \\
\hline & $\mathrm{b}$ & 0,23 & 0,41 & - & 0,76 & 0,58 & 0,37 \\
\hline Circunferência & $\mathrm{a}$ & $+0,05$ & $+0,33$ & $+0,34$ & - & $+0,33$ & $+0,18$ \\
\hline Abdominal & $\mathrm{b}$ & 0,74 & 0,02 & 0,02 & - & 0,02 & 0,22 \\
\hline \multirow[t]{2}{*}{ IMC } & $\mathrm{a}$ & $+0,10$ & $+0,28$ & $+0,30$ & $-0,05$ & $-0,09$ & - \\
\hline & $\mathrm{b}$ & 0,50 & 0,05 & 0,04 & 0,71 & 0,53 & - \\
\hline
\end{tabular}

$\mathrm{a}=$ coeficiente de correlação de Spearmann

$\mathrm{b}=\mathrm{p}$-valor

$\mathrm{Na}$ Tabela 2 são apresentados os coeficientes de correlação de postos de Spearmann. Os valores observados nas correlações da glicemia com as demais variáveis foram, em geral, menores que os da PAS.

Dada a existência das correlações já mencionadas entre idade e variáveis antropométricas, e destas entre si, decidiu-se estimar as correlações entre glicemia e variáveis antropométricas controlando-se fatores potencialmente confounding, através dos coeficientes parciais de correlação de Spearmann. Na Tabela 5 são apresentados os resultados mais relevantes.

Ao se controlar a variável peso, a circunferência abdominal manteve-se correlacionada positivamente com a glicemia, só que de forma mais intensa. Já ao se controlar pela circunferência abdominal, o peso e o IMC tornaram mais fortes suas correlações inversas com a glicemia.

TA BELA 5. Coeficientes Parciais de Correlação de Spearmann e p-valor entre Glicemia e Medidas Antropométricas e Idade

\begin{tabular}{|c|c|c|c|c|c|c|c|}
\hline \multirow[b]{2}{*}{$\begin{array}{l}\text { Variável } \\
\text { de Controle }\end{array}$} & & \multicolumn{6}{|c|}{ Coeficientes de Correlação entre Glicemia e } \\
\hline & & Idade & Peso & Altura & $\begin{array}{c}\text { Circunferência } \\
\text { Abdominal }\end{array}$ & $\begin{array}{c}\text { Circunferência } \\
\text { do Quadril }\end{array}$ & IMC \\
\hline \multirow[t]{2}{*}{ Idade } & $\mathrm{a}$ & - & $-0,12$ & $-0,10$ & $+0,20$ & $+0,08$ & $-0,05$ \\
\hline & $\mathrm{b}$ & - & 0,43 & 0,17 & 0,17 & 0,59 & 0,75 \\
\hline \multirow[t]{2}{*}{ Peso } & $\mathrm{a}$ & $+0,17$ & - & $-0,02$ & $+0,32$ & $+0,21$ & $+0,05$ \\
\hline & $\mathrm{b}$ & 0,24 & - & 0,88 & 0,03 & 0,17 & 0,75 \\
\hline \multirow[t]{2}{*}{ Circunferência Abdominal } & a & $+0,22$ & $-0,30$ & $-0,19$ & - & $-0,20$ & $-0,29$ \\
\hline & $\mathrm{b}$ & 0,14 & 0,03 & 0,19 & - & 0,18 & 0,05 \\
\hline
\end{tabular}

$\mathrm{a}=$ coeficiente parcial de Spearmann 


\section{DISCUSSÃO}

As doenças crônico-degenerativas vêm assumindo, gradativamente, um papel importante no perfil epidemiológico brasileiro nos últimos 50 anos. Inquéritos populacionais têm encontrado elevadas prevalências de hipertensão arterial (Costa, 1981; Klein \& Araújo, 1985; Rego et al., 1990) e diabetes (Cenepi, 1992), sendo as doenças cardiovasculares a primeira causa de mortalidade no país (MS, 1988).

Ao contrário do que ocorreu com as doenças infecto-contagiosas, os avanços tecnológicos nos métodos diagnósticos e terapêuticos para as doenças crônico-degenerativas não tiveram o impacto esperado na mortalidade por este grupo de doenças nas últimas décadas (MS, 1986). Assim, é indispensável associar às novas tecnologias medidas preventivas coletivas.

A análise dos coeficientes de correlação entre PA e medidas antropométricas revelou uma média de pressão arterial mais elevada no sexo masculino, reproduzindo resultados de outros estudos, tanto com os Yanomámi (INTERSALT, 1988) como com outras populações primitivas (Hern, 1991). É interessante notar, também, que, ao contrário do que é encontrado na maior parte dos estudos sobre PA com populações não-primitivas, entre os Yanomámi não se verificou uma correlação positiva da PAS com a idade. Na verdade, nossos dados mostraram uma queda nos níveis da PAS conforme aumentava a idade. Embora tal relação possa decorrer do fato de os índios mais velhos apresentarem menor massa corporal, tal hipótese não se confirmou através de uma redução da força desta correlação, quando controlada pelo IMC.

Dustan (1991) cita estudos em populações primitivas nos quais, mesmo não havendo aumento do peso com a idade, foi possível correlacionar o peso com a PA. Estudos com africanos e sul-americanos, também citados pelo autor, mostram que a PA tende a não se elevar com a idade nestas populações, mas correlaciona-se com o peso. No Suriname, a PAS dos homens correlacionava-se negativamente com a idade e positivamente com a altura, enquanto a PAD correlacionava-se positivamente com o peso em ambos os sexos. Em nossos resultados também observamos correlações positivas entre as pressões, tanto a sistólica como a diastólica, com as variáveis peso e altura.

Em sua revisão, Dustan (1991) cita estudos realizados entre os Xavánte e os Kayapó, na Amazônia, onde foram encontrados níveis baixos de PA, além de outros, conduzidos junto aos Yanomámi e aos Waoraní, nos quais a PA não se eleva com a idade. Alguns destes estudos relacionam a dieta sem sal destas populações aos baixos níveis tensionais.

Fraser (1986), ao apresentar uma comparação das médias de PA de diferentes grupos etários, obtidas a partir de vários estudos realizados em populações primitivas, com as de outros estudos com populações urbanas, mostra que apenas estas últimas têm elevação significativa da PA com a idade. A maioria destes estudos mediu o sal ingerido (urina de 24 horas), observando que o aumento da concentração de sal na urina acompanha o grau de elevação da PA com a idade.

Em relação à obesidade, fica clara sua importância sobre a PAS quando se analisam os coeficientes de correlação parciais envolvendo as variáveis peso e altura. Assim é que, para um mesmo peso, os índios mais altos e, portanto, mais magros tiveram menores pressões. $\mathrm{Da}$ mesma forma, o controle pela variável altura manteve a correlação positiva entre PAS e peso, o que quer dizer que, para indivíduos da mesma altura, os mais pesados apresentaram níveis de PAS mais elevados.

No caso da PAD, o controle pelo peso reduziu sensivelmente sua correlação com a altura. Assim, ao contrário da PAS, parece que os índios mais magros não apresentavam menores níveis de PAD.

Considerando-se que o IMC reflete uma relação entre o peso e a altura, parece-nos consistente que a PAS tenha se correlacionado mais intensamente com este índice do que a PAD (ver Tabela 2).

A obesidade é considerada um fator de risco isolado para o diabetes (Dunn et al., 1970; West \& Kalbflerisch, 1970; Wilson et al., 1981), mas seu poder, neste aspecto, aumenta quando associada à alimentação inadequada e ao sedentarismo (King, 1988). Numerosos estudos com populações diversas, como brancos, na Suécia 
(Ohlson et al., 1985), mexicanos, nos Estados Unidos (Haffner et al., 1990), nipo-americanos (Bergstron et al., 1990), índios norte-americanos (Szathmary \& Holt, 1983), micronésios das ilhas Nauru (Balkam et al., 1985), bem como indianos, chineses e crioulos das ilhas Maurícios (Dowse et al., 1991), mostram que a gordura corporal central (com concentração abdominal) é um fator de risco para IGT (Impaired Glucose Tolerance), diabetes não-insulino-dependente e doenças cardiovasculares, independente da presença ou grandeza da obesidade (Feldmam et al., 1969; Hartz et al., 1984; Koltermen et al., 1981; Zimmet \& West, 1992). O caminho para se chegar ao diabetes passa, nestes casos, pelo desenvolvimento da hiperinsulinemia (Bergstron et al., 1990; Dowse et al., 1991).

A análise dos dados antropométricos da população Yanomámi por nós estudada mostrou que as mulheres têm menores médias de altura e peso que os homens, assim como um menor IMC. Apesar disso, apresentam maior média de circunferência abdominal, o que caracteriza uma forma de distribuição de gordura mais central.

A média de glicemia foi mais elevada no sexo feminino do que no masculino. Na literatura, a distribuição de diabetes segundo o sexo não apresenta um padrão homogêneo. Em Cingapura (Thai, 1987) e na Austrália (Cameron et al., 1986) também ocorreu um aumento da prevalência de diabetes devido à aculturação, havendo um predomínio no sexo masculino. Já entre os índios Pima, nos Estados Unidos (Knowler et al., 1978) e Mapuche, no Chile (Larenas et al., 1985), a maior proporção verifica-se no sexo feminino, com valores semelhantes entre as populações nativas de Fiji (Zimmet, 1983) e Nauru (Zimmet et al., 1984).

A prevalência de IGT varia amplamente entre as populações, sendo que a mesma não é necessariamente predita pela taxa de diabetes na mesma área (Dowse et al., 1991). Entre aborígines australianos urbanizados podem ser observadas elevadas prevalências de diabetes nãoinsulino-dependente $(16,7 \%$ e $14,6 \%$ para homens e mulheres, respectivamente), embora, comparativamente, com baixas prevalências de IGT - respectivamente de $0,7 \%$ e $4,2 \%$ para homens e mulheres (Cameron et al., 1986), contrastando com outro estudo conduzido em uma zona rural das ilhas Fiji, onde a prevalência de IGT é 3,5 vezes maior que a de diabetes não-insulino-dependente (Zimmet, 1983). Ainda, entre os montanheses da Papua Nova Guiné não foi detectado diabetes, embora tenham sido observados 3,5\% de IGT entre os homens e $1,2 \%$ entre as mulheres (King, 1984).

Os coeficientes parciais de correlação de Spearmann apontam para algumas possíveis explicações para os achados de glicemia. A análise das correlações entre glicemia e variáveis antropométricas, controlando-se o efeito do peso, mostrou que a circunferência abdominal correlacionou-se positivamente com os níveis de glicose capilar. Isto significa que, entre índios com o mesmo peso, aqueles que apresentavam maior circunferência abdominal tinham níveis glicêmicos mais elevados. Por outro lado, ao se controlar a variável circunferência abdominal, o peso e o IMC se mostraram-se inversamente correlacionados com a glicemia. Isto quer dizer que, entre índios com a mesma circunferência abdominal, aqueles com maiores peso e IMC, e, portanto, com menor acúmulo de gordura no abdome, apresentavam níveis de glicemia mais baixos. Tais achados são compatíveis com a idéia de que, dentre as variáveis estudadas, a concentração de gordura no abdome parece ser a mais importante para explicar a variação da glicemia no grupo estudado. Assim sendo, pode-se supor que um dos motivos pelos quais as mulheres tenham tido níveis mais elevados de glicemia advenha do fato de estas possuírem maior média de circunferência abdominal.

Ao se investigar a relação tanto da pressão arterial quanto da glicemia com variáveis antropométricas, não se pode desconsiderar a existência de controvérsias quanto à utilização de índices de obesidade. No caso específico da população por nós estudada, a razão $\mathrm{CA} / \mathrm{CQ}$ parece não ser um bom índice, pois, ao contrário do peso e do IMC, este índice correlacionou-se fracamente tanto com a glicemia quanto com a pressão arterial. Uma possível explicação para isso seria a forte influência da conformação corporal sobre esta razão, pois dois indivíduos podem ter o mesmo valor para a razão $\mathrm{CA} / \mathrm{CQ}$ por motivos diferentes: ou por uma grande circunferência abdominal (obeso) ou por uma pequena circunferência de quadril (sem ser obeso). 


\section{CONCLUSÃO}

Os resultados encontrados mostram que os Yanomámi, assim como ocorreu com outras populações primitivas, podem, superada a atual fase de predomínio de doenças infecto-contagiosas e mantendo-se a tendência à sedentarização e mudança do padrão alimentar, vir a apresentar quadros mórbidos de doenças crônico-degenerativas. A prevalência ainda baixa destas doenças entre eles oferece uma oportunidade única de prevenção. A preservação das características culturais deste povo parece ser a melhor forma de se evitar a proliferação dos principais fatores de risco: a obesidade (em especial a centrípeta), a sedentarização e a introdução de produtos alimentícios industrializados.

\section{AGRADECIMENTOS}

Agradecemos à equipe de saúde do Distrito Sanitário Yanomámi/Boa Vista - Roraima.

\section{RESUMO}

BLOCH, K. V.; COUTINHO, E. S. F.; LÔBO, M. E. C.; OLIVEIRA, J. E. P. \& MILECH, A. Pressão Arterial, Glicemia Capilar e Medidas Antropométricas em uma População Yanomámi. Cad. Saúde Públ., Rio de Janeiro, 9 (4): 428-438, out/dez, 1993.

Com o objetivo de avaliar o comportamento da glicemia capilar e das pressões arteriais sistólica (PAS) e diastólica (PAD) em função de medidas antropométricas, foram examinados 72 Yanomámi adultos na Área de Relações Intercomunitárias de Surucucus, Estado de Roraima.

A pressão arterial foi mais elevada nos homens do que nas mulheres (PAS $=109,8$ $\mathrm{mm} \mathrm{Hg}$ e $100,2 \mathrm{~mm} \mathrm{Hg}$; PAD = 71,2 $\mathrm{mm} \mathrm{Hg}$ e $63,5 \mathrm{~mm} \mathrm{Hg}$, respectivamente). Não houve valores compatíveis com a hipertensão arterial. A PAS correlacionou-se negativamente com a idade e positivamente com altura, peso, índice de massa corporal (IMC) e circunferências abdominal e do quadril. O controle pelo IMC não alterou a correlação inversa entre PAS e idade. A correlação da PAS com a altura inverteu-se quando controlada pelo peso, enquanto o controle pela altura não alterou a correlação positiva entre peso e PAS. A PAD correlacionou-se positivamente com o peso e a altura.

A glicemia foi significativamente mais elevada nas mulheres do que nos homens (114,1 mg/dl e 98,4 mg/dl, respectivamente). Controlando-se pelo peso, a circunferência abdominal correlacionou-se positivamente com a glicemia. Ao se controlar pela circunferência abdominal, o peso e o IMC correlacionaram-se inversamente com a glicemia.

Os achados sugerem que, enquanto a PAS correlaciona-se principalmente com a massa corporal, a glicemia capilar está mais correlacionada com a concentração abdominal de gordura.

Palavras-Chave: Pressão Arterial; Glicemia; Antropometria; Yanomámi; Índios

\section{REFERÊNCIAS BIBLIOGRÁFICAS}

ALBERT, B., 1985. Temps du Sang, Temps des Cendres. Representation de la Maladie, Systeme Rituel et Espace Politique chez les Yanomámi du Sud-Est (A mazonie Brésilienne). Tese de Doutorado, Paris: Universidade de Paris $X$ - Nature.

BALKAM, B.; KING, H.; ZIMMET, P. \& RAPER, L. R., 1985. Factors associated with the development of diabetes in the Micronesian population of Nauru. American Journal of Epidemiology, 122: 594-605.

BERGSTRON, R. W.; NEWELL-MORRIS, L. L.; LEONETTI, D. I.; SCHUMAN, W. P. \& FUGIMOTO, W. Y., 1990. Association of elevated fasting G-peptide level and increased intra-abdominal fat distribution with development of NIDDM in Japanese-American mem. Diabetes, 39: 104-111.

CAMERON, W. I.; MOFFIT, P. S. \& WILLIAMS, D. R. R., 1986. Diabetes Mellitus in Australian Aborigines of Bouke, New South Wales. Diabetes Research in Clinics Practice, 2: 307-314.

CENEPI (Comissão Coordenadora Central do Estudo Multicêntrico da Prevalência do Diabetes Mellitus no Brasil), 1992. Estudo multicêntrico sobre a prevalência do diabetes mellitus no Brasil. Informe Epidemiológico do SUS, 3: 45-73. 
COSTA, E. A., 1981. A Cross-Sectional Survey of Blood Pressure in Rio Grande do Sul, Brazil. Ph.D. Dissertation, Londres: London University.

DOWSE, G. K.; ZIMMET, P.; GAREEBOO, H.; ALBERTI, K. G. M. N.; TUONILEHTO, J.; FINCH, C. F.; CHITSON, P. \& TUISIDAS, H., 1991. Abdominal obesity and physical inactivity are risk factors for NIDDM and impaired glucose tolerance in Indian, Creole, and Chinese Mauritians. Diabetes Care, 14: 271-282.

DUNN, J. P.; IPSEN, J.; ELSOM, K. O. \& OHTANI, M., 1970. Risk factors in coronary artery disease, hypertension and diabetes. American Journal of M edical Science, 259: 309-322.

DUSTAN, H. P., 1991. Hypertension and obesity. Primary Care, 18: 495-507.

FELDMAN, R.; SENDER, A. J. \& SIEGLAMB, A. B., 1969. Differences in diabetic and non-diabetic fat distribution patterns by skinfold measurements. Diabetes, 18: 478-486.

FRASER, G. E., 1986. Preventive Cardiology. New York: Oxford University Press.

HAFFNER, S. N.; STERN, M. P.; MITCHELL, B. D.; HAZUDA, H. P. \& PATTERSON, J. K., 1990. Incidence of type II diabetes in Mexican Americans predicted by fasting insulin and glucose levels, obesity and body fat distribution. Diabetes, 39: 283-288.

HARTZ, A. J.; RUPLEY, D. C. \& RIMM, A. A., 1984. The association of girth measurements with disease in 32,856 women. American Journal of Epidemiology, 119: 71-80.

HERN, W. M., 1991. Health and demography of native Amazonians: Historical perspective and current status. Cadernos de Saúde Pública, 7: 451-480.

INTERSALT, 1988. INTERSALT: An international study of electrolyte excretion and blood pressure. Results for 24-hour urinary sodium and potassium excretion. British Medical Journal, 297: 319-328.

JOINT NATIONAL COMMITTEE, 1988. The 1988 Report of The Joint National Committee on detection, evaluation, and treatment of high blood pressure. Archives of Internal Medicine, 148: 1023-1038.

KING, H., 1984. Glucose tolerance in a highland population in Papua New Guinea. Diabetes Research, 1: 45-51.

, 1988. Aetiology. Baillierrs Clinics in

Epidemiology and M etabolism, 2: 291-305.
KLEIN, C. H. \& ARAÚJO, J. W. G., 1985. Fumo, bebida alcoólica, migração, instrução, ocupação, agregação familiar e pressão arterial em Volta Redonda, Rio de Janeiro. Cadernos de Saúde Pública, 1: 160-176.

KNOWLER, W. C.; BENNETT, P.; HAMMAN, R. F. \& MILLER, M., 1978. Diabetes incidence and prevalence in Pima Indians. A merican Journal of Epidemiology, 108: 497-505.

KOLTERMEN, O. G.; GRAY, R. S.; GRIFFIN, J.; BURSTEIN, P.; INSEL, J.; SCARLETT, J. A. \& OLEFSKY, J. M., 1981. Receptor and post-receptor defects contribute to the insulin resistence in non-insulin dependent diabetes mellitus. Journal of Clinical Investigation, 68: 957-969.

LARENAS, G.; CABRERA, H. \& LARUSSO, B., 1985. Prevalence of diabetes in indigenous (Mapuche) community in the IX Region in Chile. Revista M edica de Chile, 113: 1121-1125.

MACULAY, A. C.; MONTOUR, L. T. \& ADELSON, N., 1988. Prevalence of diabetic and atherosclerotic complications among Mohawk Indians of Kahnawake, P.Q. Canadian Medical Association Journal, 139: 221-224.

MENEGOLA, I. A. \& LÔBO, M. S. C., 1991. Primeiro Relatório do Distrito Sanitário Yanomámi: Avaliação das Atividades e Diagnóstico de Saúde. Boa Vista: Fundação Nacional da Saúde, Coordenação Regional de Roraima. (Mimeo.)

MS (Ministério da Saúde); DNDCD (Divisão Nacional de Doenças Crônico-degenerativas) \& SNPES (Secretaria Nacional de Programas Especiais de Saúde), 1986. Controle das Doenças Não Transmissíveis no Brasil. Brasília: Coordenadoria de Comunicação Social do Ministério da Saúde. (Mimeo.)

MS (Ministério da Saúde); DNDCD (Divisão Nacional de Doenças Crônico-degenerativas); SNPES (Secretaria Nacional de Programas Especiais de Saúde) \& INAMPS (Instituto Nacional de Assistência Médica da Previdência Social), 1988. Doenças Crônico-Degenerativas: evolução e tendências atuais - I. Brasília: Coordenadoria de Ciência e Tecnologia. (Mimeo.)

MONTOUR, L. T. \& MACAUSAY, A. C., 1988. Diabetes mellitus and atherosclerosis: returning research results to the Mohawk community. Canadian Medical Association Journal, 139: 201-202.

MUSTO, R. J., 1990. Indian reserves: Canada's developing nations. Canadian Family Physician, 36: $105-108$. 
OHLSON, L. O.; LARSSON, B.; SVARDSUDD, K.; WELLIN, L.; ERIKSSON, H.; WILHELMSEN, L.; BJORNTORP, P. \& TIBBLIN, G., 1985. The influence of body fat distribution on the incidence of diabetes mellitus: 13,5 years of followup of the participants in the study of men born in 1913. Diabetes, 34: 1055-1058.

OMS (Organização Mundial da Saúde), 1978. Hipertensión Arterial. Informe de un Comité de Expertos de la OMS. Geneva: OMS. (Série de Informes Técnicos, 628)

PITHAN, O. A.; VARGA, I.; RAMOS, A. R. \& ALBERT, B., 1991a. Urihi: Terra, Economia e Saúde Yanomámi. Brasília: ORSTOM/Universidade de Brasília. (Série Antropológica, 119)

PITHAN, O. A.; CONFALONIERI, U. E. C. \& MORGADO, A. F., 1991b. A situação de saúde dos índios Yanomámi: Diagnóstico a partir da Casa do Índio de Boa Vista, Roraima, 1987 1989. Cadernos de Saúde Pública, 7: 563-580.

REGO, R. A.; BERARDO, F. A. N.; RODRIGUES, S. S. R.; OLIVEIRA, Z. M. A.; OLIVEIRA, M. B.; VASCONCELLOS, C.; AVENTURATO, L. V. O.; MONCAU, J. E. C. \& RAMOS, L. R., 1990. Fatores de risco para doenças crônicas não-transmissíveis: Inquérito domiciliar no município de São Paulo, SP (Brasil). Metodologia e resultados preliminares. Revista de Saúde Pública, 24: 277-285.

SIEGEL, S., 1981. Estatística Não-Paramétrica. Rio de Janeiro: Editora McGraw-Hill do Brasil.

SZATHMARY, E. J. E. \& HOLT, N., 1983. Hyperglicemia in dogrib Indians of Northwest Territories, Canadá: Association with age and centripetal distribution of body fat. Human Biology, 55: 493-515.

THAI, A. C., 1987. Changing prevalence of diabetes mellitus in Singapore over a ten-year period. In: Epidemiology of Diabetes Mellitus: Proceedings of International Symposium on Epidemiology of Diabetes M ellitus (S. Vannasaeng, W. Natitiant \& F. Chandrapraset, eds.), pp. 63-67, Bangkok: Crystal House Press.
WEST, K. M. \& KALBFLERISCH, J. M., 1970. Diabetes in Central America. Diabetes, 19: 656-663.

WILSON, P. W.; McGEE, D. L. \& KAMMEL, W.B., 1981. Obesity, VLDL and glucose intolerance over fourteen years: The Framingham Study. A merican Journal of Epidemiology, 114: 697-704.

WHO (World Health Organization), 1985: Diabetes mellitus. Report of a Who study group. Geneva: WHO (Technical Report Series, 727)

ZIMMET, P.; DOWSE, G.; SERJEANTSON, S.; FINCH, C. \& KING, H., 1990. The epidemiology and natural history of NIDDM - Lesson from the South Pacific. Diabetes M etabolism Review, 6: 91-124.

ZIMMET, P., 1983. Prevalence of diabetes and impaired glucose tolerance in the biracial (Melanesian and Indian) population of Fiji: a rural-urban comparison. A merican Journal of Epidemiology, 118: 673-688.

ZIMMET, P.; KING, H.; TAYLOR, L. R.; BALKAU, B.; BORGER, J.; HERIOT, W. \& THOMA, K., 1984: The high prevalence of diabetes mellitus, impaired glucose tolerance and diabetic retinopathy in Nauru — The 1982 survey. Diabetes Research, 1: 13-18.

ZIMMET, P. \& WEST, K., 1992. Lecture 1991 challenges in diabetes epidemiology. From west to the rest. Diabetes Care, 15: 232-253. 